\title{
Performance Analysis of Spatial Modulation
}

\author{
(Invited Paper) \\ Marco Di Renzo ${ }^{(1)}$ and Harald Haas ${ }^{(2)}$ \\ (1) L2S, UMR 8506 CNRS - SUPELEC - Univ Paris-Sud \\ Laboratory of Signals and Systems (L2S), French National Center for Scientific Research (CNRS) \\ École Supérieure d'Électricité (SUPÉLEC), University of Paris-Sud XI (UPS) \\ 3 rue Joliot-Curie, 91192 Gif-sur-Yvette (Paris), France \\ (2) The University of Edinburgh, College of Science and Engineering \\ School of Engineering, Institute for Digital Communications (IDCOM) \\ Joint Research Institute for Signal and Image Processing \\ Mayfield Road, Edinburgh, EH9 3JL, United Kingdom (UK) \\ E-Mail: marco.direnzo@lss.supelec.fr, h.haas@ed.ac.uk
}

\begin{abstract}
In this paper, we propose a framework for analyzing the performance of multiple-antenna wireless systems using the recently proposed Spatial Modulation (SM). More specifically, we derive upper bounds for computing the Average Bit Error Probability (ABEP) of Multiple-Input-Single-Output (MISO) systems over general fading channels. The framework accounts for arbitrary modulation schemes, and can handle both independent and correlated channels with arbitrary fading parameters. Monte Carlo simulations are shown to substantiate the proposed analytical derivation. Furthermore, the performance of SM is compared to Multilevel Phase Shift Keying ( $M$-PSK) modulation, and it is shown that SM can offer the same spectral efficiency with a smaller bit error probability.

Index Terms-Spatial Modulation (SM), performance analysis.
\end{abstract}

\section{INTRODUCTION}

Since the late '90s, multiple-antenna techniques have been recognized as a key technology for modern wireless communications, which provide new degrees of freedom for achieving higher data rates and smaller error probabilities by exploiting the previously unused spatial domain in novel ways [1]. In particular, higher data rates can be achieved via spatial multiplexing techniques such as the V-BLAST (Vertical Bell Laboratories Layered Space-Time) scheme [2]. These techniques simultaneously transmit independent information messages over multiple transmit-antennas to achieve a spatial multiplexing gain equal.

However, the price to be paid for this increase in the data rate is additional hardware and signal processing complexity. For example, V-BLAST requires a multi-stream detector which is based on an interference-cancelation type algorithm to decode the superimposed transmitted messages whose complexity drastically increases with the number of transmitantennas. Furthermore, it is also known that multi-stream Maximum-Likelihood (ML) detectors offer a prohibitively high computational complexity, which increases exponentially with the number of transmit-antennas [1].
In the depicted context, Spatial Modulation (SM) is a recently proposed spatial multiplexing scheme for MultipleInput-Multiple-Output (MIMO) systems, which is conceived to offer a multiplexing gain with respect to single-antenna systems without requiring extra bandwidth or extra transmission power, while still retaining a single-stream receiver design for ML-optimum decoding [3]. In particular, in [3] and [4] it is shown that SM can offer a multiplexing gain, with respect to single-antenna systems, that increases logarithmically with the number of transmit-antennas along with better performance than the V-BLAST scheme, while still guaranteeing the same spectral efficiency and yielding a significant reduction in computational complexity. Furthermore, in [5] it is pointed out that SM can potentially enable a low-complexity transmitter design by avoiding multiple Radio Frequency (RF) chains. Moreover, in contrast to V-BLAST, SM does not place any restriction on the minimum number of receiveantennas, which in V-BLAST has to be greater than the number of transmit-antennas. This is particularly beneficial for mobile handsets because of the limited available space and the cost constraints for these mass market devices. All these properties and requirements make SM a very attractive MIMO scheme for many potential applications, despite the fact that $\mathrm{SM}$ is unable to achieve multiplexing gains comparable to the V-BLAST scheme (because of the logarithmic vs. linear increase of data rate with the number of transmit-antennas). However, it strikes a practically relevant trade-off between throughput/performance and complexity.

Due to its very recent inception in the research community, relevant contributions on the performance analysis of SM are quite limited. In [3], the performance of SM over independent Rayleigh fading channels is studied for a sub-optimal receiver design. In [6], the principles of trellis coding are applied, for the first time, to the spatial constellation points in SM, and it is shown that the robustness to channel correlation is significantly increased. This, in turn means, that the multipleantennas can be placed more closely to each other, which is 
particulary relevant for mobile devices and smart phones. In [4], the ML-optimum detector is derived, and its performance studied over independent Rayleigh fading channels and real signal-constellation diagrams. In [7], the framework in [3] is generalized to Nakagami- $m$ fading channels by retaining the same sub-optimum receiver design. Other frameworks are available in the literature, e.g., [5], [8] $[10]$ and references therein, but all of them deal with the performance analysis of Space Shift Keying (SSK) modulation, which is a lowcomplexity implementation of SM with a reduced multiplexing gain. In particular, [10] shows the most general analytical framework for performance analysis of SSK modulation over correlated Nakagami- $m$ fading channels. However, this framework is not directly applicable to SM.

Motivated by the lack of fundamental analytical frameworks for performance analysis of SM with ML-optimum detection, the contribution of this paper is twofold. First, we propose a tight upper bound for computing the Average Bit Error Probability (ABEP) of SM for arbitrary digital signal modulation schemes and transmit-antennas. With respect to [4], our framework is not restricted to modulation schemes with a real signal-constellation, but can be used for generic complex constellation diagrams. Furthermore, it is useful for a large set of correlated fading channel models. Finally, with respect to [10], the framework is not restricted to SSK modulation. Second, we compare the performance of SM with Multilevel Phase Shift Keying ( $M$-PSK) modulation schemes, and show that SM has the desired flexibility to provide better performance, while guaranteeing the same or a better spectral efficiency. To keep the derivation at a reasonable level, in this paper we consider a typical downlink setting where the remote handset is equipped with a single receive-antenna.

The remainder of this paper is organized as follows. In Section II, the system model is introduced. In Section III, the analytical framework for performance analysis of SM is outlined. In Section IV, numerical and simulation results are shown to substantiate the accuracy of the analytical framework and to compare SM with single-antenna multiphase modulation schemes. Finally, Section V concludes the paper.

\section{SySTEM MODEL}

We consider a generic $N_{t} \times N_{r}$ Multiple-Input-SingleOutput (MISO) system, with $N_{t}$ and $N_{r}=1$ being the number of transmit- and receive-antennas, respectively. Moreover, we assume that the transmitter can send digital information via $M$ distinct signal waveforms (i.e., the so-called signalconstellation diagram). The basic idea of SM is to map blocks of information bits into two information carrying units [3]: 1) a symbol, which is chosen from a complex signal-constellation diagram, and 2) a unique transmit-antenna index, which is chosen from the set of transmit-antennas in the antennaarray (i.e., the so-called spatial-constellation diagram). The principle working mechanism of SM is depicted in Fig. 1.

In particular, at the transmitter, the bitstream emitted by a binary source is divided into blocks containing $\log _{2}\left(N_{t}\right)+$ $\log _{2}(M)$ bits each, with $\log _{2}\left(N_{t}\right)$ and $\log _{2}(M)$ being the

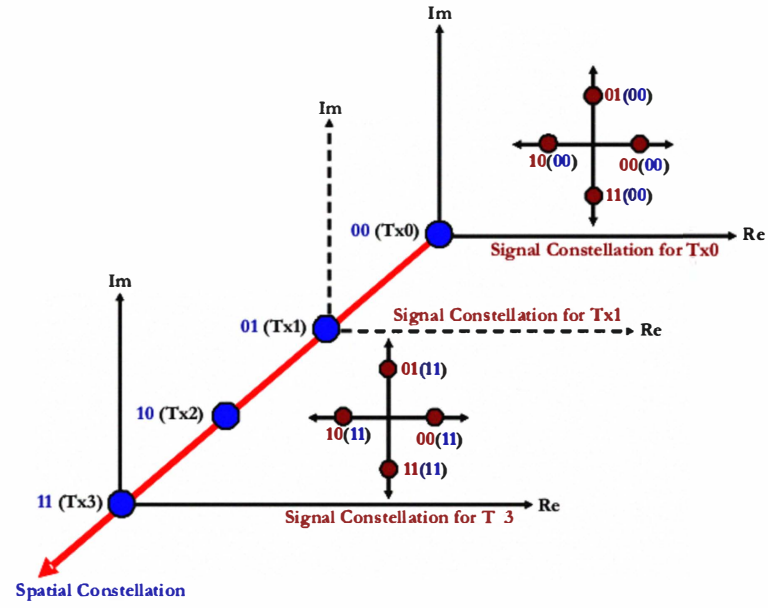

Fig. 1. Three-dimensional constellation diagram of SM: each spatialconstellation point (i.e., the antenna index) defines an independent complex plane of signal-constellation points. For illustrative purposes, only two of such planes are shown in the figure for: i) $N_{t}=4$, and ii) $M=4$. Legend: i) $\mathrm{Re}=$ real axis of the signal-constellation diagram, and ii) $\mathrm{Im}=$ imaginary axis of the signal-constellation diagram.

number of bits needed to identify a transmit-antenna in the antenna-array and a symbol in the signal-constellation diagram, respectively. Each block is split into two sub-blocks of $\log _{2}\left(N_{t}\right)$ and $\log _{2}(M)$ bits each. The bits in the first sub-block are used to select the antenna that is switched on for data transmission, while all other transmit-antennas are kept silent in the current signaling time interval. The bits in the second sub-block are used to choose a symbol in the signal-constellation diagram. The receiver solves a $N_{t} \times M-$ hypothesis detection problem to jointly estimate the transmitantenna that is not idle and the signal waveform that has been transmitted from it, which results in the estimation of the unique sequence of $\log _{2}\left(N_{t}\right)+\log _{2}(M)$ bits emitted by the encoder. Throughout this paper, we consider a ML-optimum decoder, which computes the Euclidean distance between the received signal and the set of $N_{t} \times M$ possible received signals, and chooses the closest one [4].

In this paper, the block of $\log _{2}\left(N_{t}\right)+\log _{2}(M)$ bits emitted by the encoder is called "message" and is denoted by $m_{n_{t}}$, where $n_{t}=1,2, \ldots, N_{t}$ and $m=1,2, \ldots, M$ univocally identify the active transmit-antenna and the signal waveform transmitted from it, respectively. Moreover, the related transmitted signal is denoted by $s\left(\cdot \mid m_{n_{t}}\right)$ for $n_{t}=1,2, \ldots, N_{t}$ and $m=1,2, \ldots, M$. It is implicitly assumed in this notation that, if $m_{n_{t}}$ is transmitted, the signal $s\left(\cdot \mid m_{n_{t}}\right)$ is emitted by the $n_{t}$-th transmit-antenna while all other transmit-antennas radiate no power. The $N_{t} \times M$ messages are assumed to be emitted with equal probability by the encoder.

\section{A. Notation}

Throughout this paper, we use the notation as follows. i) We adopt a complex-envelope signal representation. ii) $j=\sqrt{-1}$ is the imaginary unit. iii) $(\cdot)^{*}$ denotes the complex- 
conjugate operator. iv) $(x \otimes y)(t)=\int_{-\infty}^{+\infty} x(\xi) y(t-\xi) d \xi$ is the convolution of signals $x(\cdot)$ and $y(\cdot)$. v) $|\cdot|^{2}$ denotes square absolute value. vi) $\mathrm{E}\{\cdot\}$ is the expectation operator. vii) $\operatorname{Re}\{\cdot\}$ denotes the real part operator. viii) $\Gamma(x)=\int_{0}^{+\infty} \xi^{x-1} \exp (-\xi) d \xi$ is the Gamma function. ix) $Q(x)=(1 / \sqrt{2 \pi}) \int_{x}^{+\infty} \exp \left(-t^{2} / 2\right) d t$ is the Q-function. $\left.\mathrm{x}\right)$ $\widehat{m_{n_{t}}}$ denotes the message estimated at the receiver. xi) $E_{m}$ is the average energy transmitted by each antenna that emits a non-zero signal. xii) $T_{m}$ denotes the signaling interval for each information message. xiii) The noise $v(\cdot)$ at the input of the receive-antenna is assumed to be an Additive White Gaussian Noise (AWGN) process, with both real and imaginary parts having a double-sided power spectral density equal to $N_{0}$. xiv) For ease of notation, we set $\bar{\gamma}=E_{m} /\left(4 N_{0}\right)$. xv) $\delta(\cdot)$ is the Dirac delta function. xvi) $G_{p, q}^{m, n}\left(. \mid \begin{array}{c}\left(a_{p}\right) \\ \left(b_{q}\right)\end{array}\right)$ is the Meijer$\mathrm{G}$ function defined in [11, Ch. 8, pp. 519].

\section{B. Channel Model}

We consider the frequency-flat slowly-varying fading channel model as follows:

- $h_{n_{t}}(t)=\alpha_{n_{t}} \delta\left(t-\tau_{n_{t}}\right)$ is the channel impulse response of the transmit-receive wireless link from the $n_{t}$-th transmit-antenna to the single receive-antenna for $n_{t}=1,2, \ldots, N_{t} . \alpha_{n_{t}}=\beta_{n_{t}} \exp \left(j \varphi_{n_{t}}\right)$ and $\tau_{n_{t}}$ are the complex channel gains and time-delays over the $n_{t}$-th transmit-receive wireless link, respectively.

- The delays $\tau_{n_{t}}$ for $n_{t}=1,2, \ldots, N_{t}$ are assumed to be independent and uniformly distributed in $\left[0, T_{m}\right)$, but known at the receiver, i.e., perfect time-synchronization is considered.

- No specific distribution for the channel envelopes, $\beta_{n_{t}}$, and the channel phases, $\varphi_{n_{t}}$, for $n_{t}=1,2, \ldots, N_{t}$ is assumed. Throughout this paper, we denote by $\Omega_{n_{t}}=$ $\mathrm{E}\left\{\beta_{n_{t}}^{2}\right\}$ the mean square value of the fading envelope. In Section III, we will see that the conditional error probability computed in this paper holds for any fading distributions and fading parameters. Explicit closed-form expressions of the ABEP for independent Nakagami- $m$ fading channels are given.

\section{ML-Optimum Detector}

Let $\bar{m}_{\bar{n}_{t}}$ with $\bar{n}_{t}=1,2, \ldots, N_{t}$ and $\bar{m}=1,2, \ldots, M$ be the transmitted message ${ }^{1}$. The signal received after propagation through the wireless fading channel and impinging upon the single receive-antenna can be written as follows:

$$
r(t)=\tilde{s}\left(t \mid \bar{m}_{\bar{n}_{t}}\right)+v(t) \quad \text { if } \bar{m}_{\bar{n}_{t}} \text { is sent }
$$

where $\quad \tilde{s}\left(t \mid \bar{m}_{\bar{n}_{t}}\right)=\left(s\left(\cdot \mid \bar{m}_{\bar{n}_{t}}\right) \otimes h_{\bar{n}_{t}}\right)(t)=$ $\alpha_{\bar{n}_{t}} s\left(t-\tau_{\bar{n}_{t}} \mid \bar{m}_{\bar{n}_{t}}\right)=\beta_{\bar{n}_{t}} \exp \left(j \varphi_{\bar{n}_{t}}\right) s\left(t-\tau_{\bar{n}_{t}} \mid \bar{m}_{\bar{n}_{t}}\right)$ for $\bar{n}_{t}=1,2, \ldots, N_{t}$ and $\bar{m}=1,2, \ldots, M$.

\footnotetext{
${ }^{1}$ In order to avoid any confusion with the adopted notation, let us emphasize that indexes with overline identify the actual message that is transmitted, while indexes without overline denote the trial message that is tested by the detector to solve the $N_{t} \times M$-hypothesis detection problem.
}

In particular, (1) is a general $N_{t} \times M$-hypothesis detection problem [12, Sec. 7.1], [13, Sec. 4.2, pp. 257] in AWGN, when conditioning upon fading channel statistics. Accordingly, the ML-optimum detector with full Channel State Information (CSI) and perfect time-synchronization at the receiver is as follows [4], [12, Sec. 7.1]:

$$
\widehat{m_{n_{t}}}=\underset{\substack{m_{n_{t}} \\ \text { for } n_{t}=1,2, \ldots, N_{t} \\ \text { and } m=1,2, \ldots, M}}{\arg \max }\left\{D\left(m_{n_{t}}\right)\right\}
$$

where $D(\cdot)$ for $n_{t}=1,2, \ldots, N_{t}$ and $m=1,2, \ldots, M$ is the decision metric defined in what follows:

$$
\begin{aligned}
D\left(m_{n_{t}}\right) & =\operatorname{Re}\left\{\int_{T_{m}} r(t) \tilde{s}^{*}\left(t \mid m_{n_{t}}\right) d t\right\} \\
& -\frac{1}{2} \int_{T_{m}} \tilde{s}\left(t \mid m_{n_{t}}\right) \tilde{s}^{*}\left(t \mid m_{n_{t}}\right) d t
\end{aligned}
$$

If the transmitted message is $\bar{m}_{\bar{n}_{t}}$, which results in switching on the $\bar{n}_{t}$-th transmit-antenna and sending the signal waveform $s\left(\cdot \mid m_{n_{t}}\right)$ out from it, the detector will be successful in detecting the transmitted message, i.e., $\widehat{m}_{n_{t}}=\bar{m}_{\bar{n}_{t}}$, if and only if $\max _{\substack{n_{t}=1,2, \ldots, N_{t} \\ m=1,2, \ldots, M}}\left\{D\left(m_{n_{t}}\right)\right\}=D\left(\bar{m}_{\bar{n}_{t}}\right)$.

Conventional SM [3], [14] assumes that the signal transmitted by the $\bar{n}_{t}$-th transmit-antenna is $s\left(t \mid \bar{m}_{\bar{n}_{t}}\right)=$ $\sqrt{E_{m}} \chi_{\bar{m}} w(t)$ for $\bar{n}_{t}=1,2, \ldots, N_{t}$ and $\bar{m}=1,2, \ldots, M$, where $w(\cdot)$ denotes the unit-energy (i.e., $\int_{-\infty}^{+\infty}|w(t)|^{2} d t=1$ ) elementary pulse waveform for each transmission, and $\chi_{\bar{m}}=$ $\rho_{\bar{m}} \exp \left(j \phi_{\bar{m}}\right)$ is the complex signal waveform transmitted by the active antenna, which belongs to the signal-constellation diagram. We emphasize here that the signal-constellation diagram is not restricted to equi-energy or real signals. Finally, we note that the signal emitted by each transmit-antenna only depends on the digital signal modulation scheme and is independent of the transmit-antenna index $\bar{n}_{t}$. Advanced SM schemes have been recently proposed where each transmitantenna can send a different signal to achieve a higher diversity gain [14]. However, the analysis of these advanced schemes is beyond the scope of this paper. Accordingly, the signal impinging upon the receive-antenna is as follows:

$$
r(t)=\sqrt{E_{m}}\left[\beta_{\bar{n}_{t}} \exp \left(j \varphi_{\bar{n}_{t}}\right)\right]\left[\rho_{\bar{m}} \exp \left(j \phi_{\bar{m}}\right)\right] w(t)+v(t)
$$

We note that in (4) the channel delays $\tau_{\bar{n}_{t}}$ for $\bar{n}_{t}=$ $1,2, \ldots, N_{t}$ do not appear explicitly. This assumption is similar to [10], where, with a slight abuse of notation, the propagation delays are embedded into the channel phases. For example, this is possible when $w(\cdot)$ is a pure sinusoidal tone.

\section{PERformance ANALYSIS}

Given the ML-optimum detector in (2), the objective of this section is to compute a closed-form expression of the ABEP for arbitrary signal-constellation diagrams. This section is composed by two different parts: i) first, we show a general approach to compute an upper bound of the BEP conditioned upon fading channel statistics, and ii) second, as a case study, we provide explicit closed-form expressions of the ABEP 


$$
\mathrm{BEP} \leq \frac{1}{2\left(M N_{t}-1\right)} \sum_{n_{t}^{(1)}=1}^{N_{t}} \sum_{n_{t}^{(2)}=1}^{N_{t}} \sum_{m^{(1)}=1}^{M} \sum_{m^{(2)}=1}^{M} \operatorname{PEP}\left(m^{(1)}, n_{t}^{(1)}, m^{(2)}, n_{t}^{(2)}\right)
$$

$$
\operatorname{PEP}\left(m^{(1)}, n_{t}^{(1)}, m^{(2)}, n_{t}^{(2)}\right)=Q\left(\sqrt{\bar{\gamma} \beta_{n_{t}}^{2} \mid \rho_{m^{(2)}} \exp \left(j \phi_{m^{(2)}}\right)-\rho_{m^{(1)}} \exp \left(j \phi_{m^{(1)}}\right)^{2}}\right)
$$

$$
\operatorname{PEP}\left(m^{(1)}, n_{t}^{(1)}, m^{(2)}, n_{t}^{(2)}\right)=Q\left(\sqrt{\bar{\gamma} \rho_{m}^{2}\left|\beta_{n_{t}^{(2)}} \exp \left(j \varphi_{n_{t}^{(2)}}\right)-\beta_{n_{t}^{(1)}} \exp \left(j \varphi_{n_{t}^{(1)}}\right)\right|^{2}}\right)
$$

$$
\operatorname{PEP}\left(m^{(1)}, n_{t}^{(1)}, m^{(2)}, n_{t}^{(2)}\right)=Q\left(\sqrt{\bar{\gamma}\left|\beta_{n_{t}^{(2)}} \rho_{m^{(2)}} \exp \left(j\left(\varphi_{n_{t}^{(2)}}+\phi_{m^{(2)}}\right)\right)-\beta_{n_{t}^{(1)}} \rho_{m^{(1)}} \exp \left(j\left(\varphi_{n_{t}^{(1)}}+\phi_{m^{(1)}}\right)\right)\right|^{2}}\right)
$$

for a M-PSK signal modulation scheme over independent Nakagami- $m$ fading channels.

\section{A. ABEP for Generic Modulations and Fading Channels}

The conditional BEP can be estimated by resorting to union bound methods. In particular, in [9] we have recently proposed a tight bound for performance analysis of SSK modulation. This bound, can be generalized, with similar arguments, to SM as shown in (5) on top of this page ${ }^{2}$, where $\operatorname{PEP}\left(m^{(1)}, n_{t}^{(1)}, m^{(2)}, n_{t}^{(2)}\right)=\operatorname{PEP}\left(m_{n_{t}^{(1)}}^{(1)} \rightarrow m_{n_{t}^{(2)}}^{(2)}\right)$ is the conditional Pairwise Error Probability (PEP) of messages $m_{n_{t}^{(1)}}^{(1)}$ and $m_{n_{t}^{(2)}}^{(2)}$ for $m^{(1)}=1,2, \ldots, M, m^{(2)}=1,2, \ldots, M$, $n_{t}^{(1)}=1,2, \ldots, N_{t}$, and $n_{t}^{(2)}=1,2, \ldots, N_{t}$, i.e., the probability of detecting $m_{n_{t}^{(2)}}^{(2)}$ when, instead, $m_{n_{t}^{(1)}}^{(1)}$ is actually transmitted.

The bound in (5) requires the accurate computation of the conditional PEPs. These functions can be obtained by using analytical steps similar to, e.g., [8]-[10], and by exploiting some properties of Gaussian processes. Due to space constraints, we avoid to report the details of the analytical derivation in this paper, but summarize only the main result. In particular, it can be shown that the conditional PEPs in (5) are as follows:

1) If $n_{t}=n_{t}^{(1)}=n_{t}^{(2)}$ and $m^{(1)} \neq m^{(2)}$, i.e., in the hypothesis detection problem in (2) we are considering two different signal waveforms emitted by the same transmit-antenna, then we have (6) on top of this page.

2) If $n_{t}^{(1)} \neq n_{t}^{(2)}$ and $m=m^{(1)}=m^{(2)}$, i.e., in the hypothesis detection problem in (2) we are considering the same signal waveform emitted by two different transmit-antennas, then we have (7) on top of this page.

\footnotetext{
${ }^{2}$ To simplify the notation, it is implicitly assumed in (5) and in all fold summations shown in this paper that the event $n_{t}^{(1)}=n_{t}^{(2)}$ and $m^{(1)}=$ $m^{(2)}$, i.e., perfect detection, needs to be kept out of the summation.
}

3) If $n_{t}^{(1)} \neq n_{t}^{(2)}$ and $m^{(1)} \neq m^{(2)}$, i.e., in the hypothesis detection problem in (2) we are considering two different signal waveforms emitted by two different transmitantennas, then we have (8) on top of this page.

By carefully looking at (6)-(8), the following considerations can be made:

- (6) is the error probability of conventional digital modulation schemes with a generic complex signal-constellation diagram. As a matter of fact, the argument of the $Q$ function is the Euclidean distance between pairs of signal-constellation points. Accordingly, closed-form expressions of (6) can be obtained for several modulation schemes, e.g., $M$-PSK or Multilevel Quadrature Amplitude Modulation ( $M$-QAM) [12, Ch. 8].

- (7) is the error probability of an equivalent SSK modulation scheme, which completely depends on channel statistics. Closed-form expressions for (7) can be found in recent literature for various channel models, e.g., in [8], [9], [10] the performance of correlated Rician, correlated Rayleigh, and correlated Nakagami- $m$ fading channels can be found.

- (8) depends on mixture contributions, which encompass both signal- and spatial-constellation diagrams. To the best of the authors knowledge, no closed-form results for general digital modulation schemes and fading channel models exist in the literature for (8). However, in several circumstances, the channel phases $\varphi_{n_{t}^{(1)}}$ and $\varphi_{n_{t}^{(2)}}$ for $n_{t}^{(1)}=1,2, \ldots, N_{t}$ and $n_{t}^{(2)}=1,2, \ldots, N_{t}$ can be assumed to be independent and uniformly distributed in $[0,2 \pi)$. In such a case, it is simple to show that $\varphi_{n_{t}^{(1)}}+\phi_{m^{(1)}}$ and $\varphi_{n_{t}^{(2)}}+\phi_{m^{(2)}}$ for $n_{t}^{(1)}=1,2, \ldots, N_{t}$, $n_{t}^{(2)}=1,2, \ldots, N_{t}, m^{(1)}=1,2, \ldots, M$, and $m^{(2)}=$ $1,2, \ldots, M$ are independent and uniformly distributed in $[0,2 \pi)$ too. Accordingly, (8) can be computed by using the same analytical frameworks used to compute (7), but replacing the mean square values $\Omega_{n_{t}^{(1)}}$ and 


$$
\mathrm{ABEP}=\mathrm{E}\{\mathrm{BEP}\} \leq \frac{1}{2\left(M N_{t}-1\right)} \sum_{n_{t}^{(1)}=1}^{N_{t}} \sum_{n_{t}^{(2)}=1}^{N_{t}} \sum_{m^{(1)}=1}^{M} \sum_{m^{(2)}=1}^{M} \mathrm{E}\left\{\operatorname{PEP}\left(m^{(1)}, n_{t}^{(1)}, m^{(2)}, n_{t}^{(2)}\right)\right\}
$$

$$
\left.\mathrm{ABEP} \leq \frac{1}{2\left(M N_{t}-1\right)} \sum_{n_{t}^{(1)}=1}^{N_{t}} \sum_{n_{t}^{(2)} \neq n_{t}^{(1)}=1}^{N_{t}} \sum_{m^{(1)}=1}^{M} \sum_{m^{(2)}=1}^{M} \mathrm{E}\left\{\operatorname{PEP}\left(m^{(1)}, n_{t}^{(1)}, m^{(2)}, n_{t}^{(2)}\right)\right\}+\frac{M}{2\left(M N_{t}-1\right)} \sum_{n_{t}=1}^{N_{t}} \operatorname{E}_{\operatorname{SEP}_{M}}\left(n_{t}\right)\right\}
$$

$$
\begin{aligned}
\mathrm{ABEP} & \leq \frac{M^{2}}{2\left(M N_{t}-1\right)} \sum_{n_{t}^{(1)}=1}^{N_{t}} \sum_{n_{t}^{(2)} \neq n_{t}^{(1)}=1}^{N_{t}}\left[\frac{1}{\pi} \int_{0}^{\pi / 2} M_{\mathrm{SSK}}\left(\frac{\bar{\gamma}}{2 \sin ^{2}(\theta)} ; n_{t}^{(1)}, n_{t}^{(2)}\right) d \theta\right] \\
& +\frac{M}{2\left(M N_{t}-1\right)} \sum_{n_{t}=1}^{N_{t}}\left[\frac{1}{\pi} \int_{0}^{\pi(M-1) / M} M_{\mathrm{PSK}}\left(\frac{2 \bar{\gamma} \sin ^{2}\left(\frac{\pi}{M}\right)}{\sin ^{2}(\theta)} ; n_{t}\right) d \theta\right]
\end{aligned}
$$

$\Omega_{n_{t}^{(2)}}$ with $\tilde{\Omega}_{n_{t}^{(1)}}=\rho_{m^{(1)}}^{2} \Omega_{n_{t}^{(1)}}$ and $\tilde{\Omega}_{n_{t}^{(2)}}=\rho_{m^{(2)}}^{2} \Omega_{n_{t}^{(2)}}$, respectively.

Finally, the ABEP over fading channel statistics can be formally computed as shown in (9) on top of this page, and closed-form expressions of the average PEPs can be obtained by exploiting the considerations mentioned above for estimating (6)-(8).

1) A More Accurate Bound: By carefully looking at (5) and (9), we can notice that the summation of all terms such that $n_{t}^{(1)}=n_{t}^{(2)}$ and $m^{(1)} \neq m^{(2)}$ gives the well-known union bound of the conventional digital signal modulation scheme used at the transmitter [12], [13]. However, there are several modulation schemes for which exact closed-form expressions of the (average) Symbol Error Probability (SEP) can be obtained [12, Ch. 8]. Moving from this consideration, we propose a more accurate bound for the ABEP in (9), which avoids union bound methods for those terms arising from the signal-constellation diagram only. More specifically, the ABEP in (9) can be re-written as shown in (10) on top of this page. In (10), $\operatorname{SEP}_{M}(\cdot)$ denotes the SEP of the digital signal modulation used at the transmitter, which can be found in [12, Ch. 8] for several modulation schemes.

\section{B. ABEP of M-PSK Modulation over Independent Nakagami-m Fading Channels}

In this section, we provide an example to show how the average PEP and SEP in (10) can be computed in closed-form for a simple setup with $M$-PSK modulation and Nakagami$m$ fading over the transmit-receive wireless links. We have decided to considering $M$-PSK modulation because for equienergy signal-constellation diagrams the final formulas in (10) and (6)-(8) yield several common terms which can be grouped together. Due to space constraints, we omit the details of the analytical derivation and report only the final result. In particular, from [10, Eq. (20)], [12, Sec. 8.1.1.3], and some algebraic manipulations, the final result in (11) on top of this page can be obtained, where $M_{\mathrm{SSK}}\left(\cdot ; n_{t}^{(1)}, n_{t}^{(2)}\right)$ and $M_{\mathrm{PSK}}\left(\cdot ; n_{t}\right)$ are as follows:

$$
\begin{aligned}
& M_{\mathrm{SSK}}\left(s ; n_{t}^{(1)}, n_{t}^{(2)}\right)=\frac{A_{1} A_{2}}{4}\left(s+B_{1}\right)^{-\left(\frac{1}{2}+\frac{C_{1}}{2}\right)}\left(s+B_{2}\right)^{-\left(\frac{1}{2}+\frac{C_{2}}{2}\right)} \\
& \times G_{2,2}^{1,2}\left(-\frac{s^{2}}{\left(s+B_{1}\right)\left(s+B_{2}\right)} \mid \begin{array}{cc}
\frac{1}{2}-\frac{C_{2}}{2} & \frac{1}{2}-\frac{C_{1}}{2} \\
0 & 0
\end{array}\right) \\
& M_{\mathrm{PSK}}\left(s ; n_{t}\right)=\left(1+\frac{\Omega_{n_{t}}}{\eta_{n_{t}}^{\mathrm{Nak}}} s\right)^{-\eta_{n_{t}}^{\mathrm{Nak}}}
\end{aligned}
$$

where $A_{i}=\left[2\left(\eta_{n_{t}^{(i)}}^{\mathrm{Nak}}\right)^{\eta_{n_{t}^{(i)}}^{\mathrm{Nak}}}\right] /\left[\Omega_{n_{t}^{(i)}}^{\eta_{n_{t}^{(i)}}^{\mathrm{Nak}}} \Gamma\left(\eta_{n_{t}^{(i)}}^{\mathrm{Nak}}\right)\right], B_{i}=$ $\eta_{n_{t}^{(i)}}^{\mathrm{Nak}} / \Omega_{n_{t}^{(i)}}, C_{i}=2 \eta_{n_{t}^{(i)}}^{\mathrm{Nak}}-1$ for $i=1,2$, and $\eta_{n_{t}}^{\mathrm{Nak}}$ is the Nakagami- $m$ fading parameter over the wireless link between the $n_{t}$-th transmit-antenna and the single receive-antenna.

Finally, we note that the integrals in (12) and (13) can be readily computed via simple numerical integration techniques. The accuracy of the bound in (11) is analyzed in Section IV for various system setups.

\section{Numerical and Simulation Results}

The aim of this section is twofold: i) analyze the accuracy of the bound in (11) for various combinations of transmitantennas, $N_{t}$, and modulation orders, $M$; and ii) compare the performance of SM with conventional $M$-PSK modulation over fading channels. For illustrative purposes, the following setup is analyzed: i) we consider independent and identically distributed Nakagami- $m$ fading, with parameters $\Omega_{n_{t}}=1$ and $\eta_{n_{t}}^{\mathrm{Nak}}=1$ for $n_{t}=1,2, \ldots, N_{t}$, over all wireless links; ii) $M \in\{2,8,32,128\}$; and iii) $N_{t} \in\{8,32\}$.

With regard to the number of transmit-antennas, $N_{t}$, used in our simulations, we have to make an important comment. On the one hand, the setup with $N_{t}=8$ is certainly feasible in a downlink setting, and requires a Base Station (BS) equipped with $N_{t}=8$ transmit-antennas which are sufficiently far apart 


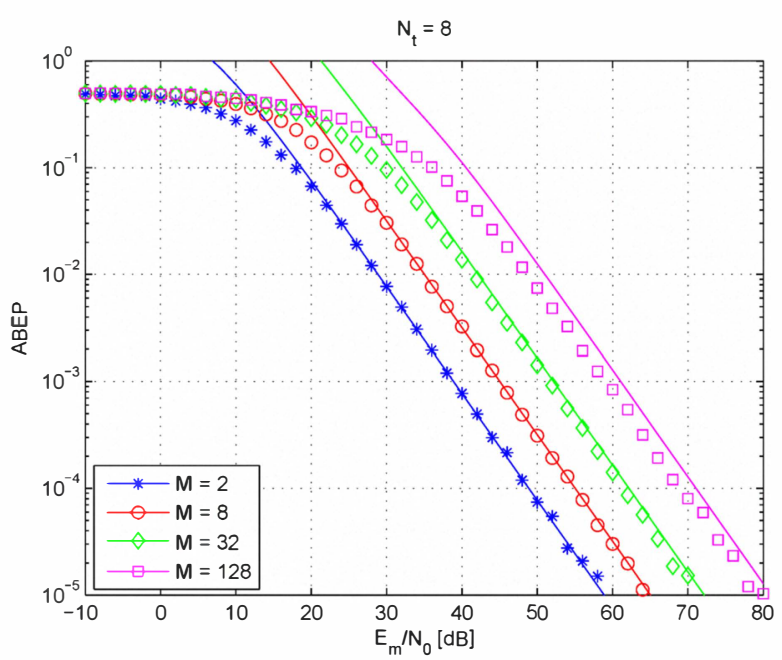

Fig. 2. ABEP against the Signal-to-Noise-Ratio (SNR) $\mathrm{E}_{\mathrm{m}} / \mathrm{N}_{0}$ for $N_{t}=8$. Markers denote Monte Carlo simulation and solid lines the bound in (11).
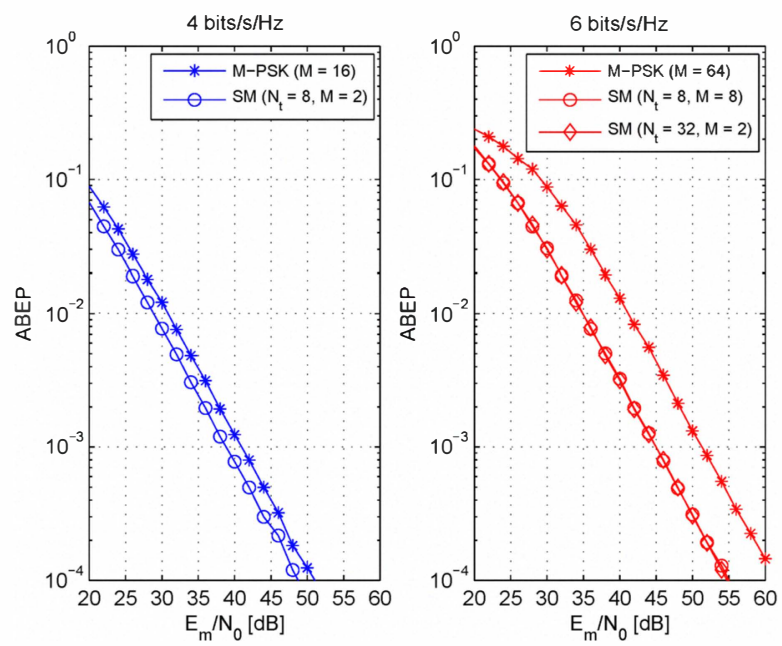

Fig. 4. ABEP against the Signal-to-Noise-Ratio (SNR) $\mathrm{E}_{\mathrm{m}} / \mathrm{N}_{0}$. Comparison between $M$-PSK modulation and SM for system setups offering the same spectral efficiency ( $4 \mathrm{bits} / \mathrm{s} / \mathrm{Hz}$ and $6 \mathrm{bits} / \mathrm{s} / \mathrm{Hz}$ ). Only Monte Carlo simulations are shown to improve the readability.

from each other to guarantee independent fading. On the other hand, the setup with $N_{t}=32$ seems to be very difficult to be realized in practice if we assume that all transmitantennas are co-located (i.e., electrically connected) in the same BS. However, in this paper we have an extended scenario in mind with respect to the typical one considered for SM [10]. We assume that the $N_{t}$ transmit-antennas are not necessarily co-located in the same BS, but can be distributed in space. This scenario builds upon a concept, which in literature is described with different terms such as, just to cite a few, JOint transmission and detection Integrated NeTwork (JOINT) [15], virtual MIMO system [16], BS cooperation [17], and Coordinated Multi-Point (CoMP) transmission scheme [18], [19]. The basic idea is that $N_{t_{1}}$ BSs, each one equipped with $N_{t_{2}}$ antennas such that $N_{t}=N_{t_{1}} N_{t_{2}}$, share their antennaarrays to allow the realization of a distributed or virtual implementation of SM. The $N_{t_{1}}$ BSs are assumed to be

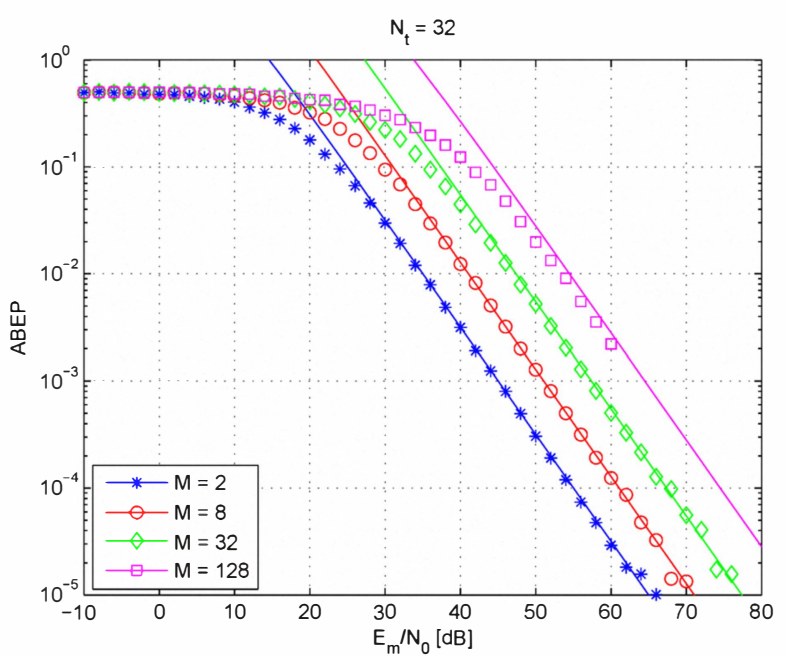

Fig. 3. ABEP against the Signal-to-Noise-Ratio (SNR) $\mathrm{E}_{\mathrm{m}} / \mathrm{N}_{0}$ for $N_{t}=$ 32. Markers denote Monte Carlo simulation and solid lines the bound in (11).
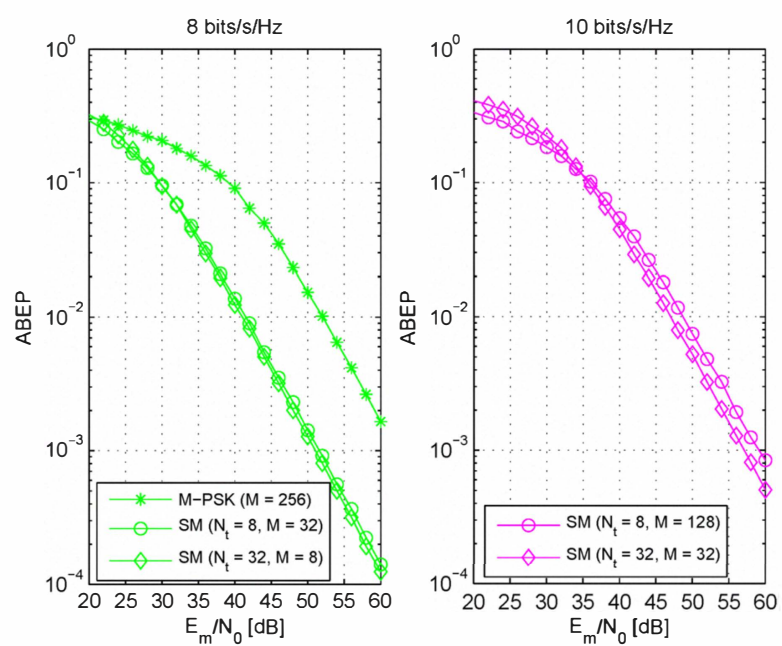

Fig. 5. ABEP against the Signal-to-Noise-Ratio (SNR) $\mathrm{E}_{\mathrm{m}} / \mathrm{N}_{0}$. Comparison between $M$-PSK modulation and SM for system setups offering the same spectral efficiency $(8 \mathrm{bits} / \mathrm{s} / \mathrm{Hz}$ and $10 \mathrm{bits} / \mathrm{s} / \mathrm{Hz})$. Only Monte Carlo simulations are shown to improve the readability.

connected via a reliable wired backhaul link, such that all of them can receive the message that the core network is intended to transmit to the remote handset. In this scenario, each antenna of the virtual antenna-array system distributed among the BSs is identified by a unique sequence of $\log _{2}\left(N_{t}\right)=$ $\log _{2}\left(N_{t_{1}} N_{t_{2}}\right)=\log _{2}\left(N_{t_{1}}\right)+\log _{2}\left(N_{t_{2}}\right)$ bits, such that the basic principle of SM can be retained: for each transmitted message only a single antenna of the virtual MIMO system is active for data transmission. With respect to conventional BS cooperation methods [18], in our distributed implementation of SM the backhaul has less stringent requirements since the cooperative BSs do not have to exchange data for cooperative beamforming, but the backhaul is used only for disseminating the information from the core network to the BSs. Furthermore, we emphasize that since the cooperative BSs do not perform distributed beamforming, no transmit-CSI is required in our scheme. With this idea in mind, the setup with $N_{t}=32$ 
transmit-antennas could be readily achieved with $N_{t_{1}}=4$ cooperative BSs each one equipped with $N_{t_{2}}=8$ transmitantennas.

Let us now analyze the numerical results shown in Figs. 25. In Figs. 2, 3, we observe that the bound introduced in (11) is quite accurate for various combinations of $N_{t}$ and $M$. As expected, we notice that the ABEP gets worse for increasing values of either $N_{t}$ or $M$. However, it can be seen that the larger either $N_{t}$ or $M$, the higher the multiplexing gain. As a consequence, there is a trade-off between achievable performance and throughput. In Figs. 4, 5, we compare the performance of $M$-PSK and SM for various target spectral efficiencies. We notice that SM always outperforms $M$-PSK, and the performance gain increases for increasing values of the target throughput. Interestingly, in Fig. 5 we observe that the performance of SM for $10 \mathrm{bits} / \mathrm{s} / \mathrm{Hz}$ is better than the performance of $M-\mathrm{PSK}$ for $8 \mathrm{bits} / \mathrm{s} / \mathrm{Hz}$ : in this case SM can offer better throughput and performance at the same time. This result is achieved because SM allows us to use low-order (i.e., $M$ is small) signal-modulation schemes by exploiting: i) the spatial domain as an additional dimension to conveying information, and ii) the randomness of the wireless channel to get a spatial-constellation diagram with points (i.e., the channel impulse responses) located further apart than the modulation points in the signal-constellation diagram. Finally, we observe that, for the considered channel model, the solutions with $N_{t}=8$ and $N_{t}=32$ offer, for the same spectral efficiency, almost the same performance. The reason is simple: by assuming an independent and identically distributed fading channel model there is no difference if the transmitantennas are either co-located in the same BS or distributed in multiple BSs. The analysis of more realistic channel models taking into account the spatial positions of the BSs is being our current research activity.

\section{CONCLUSION}

In this paper, we have proposed an accurate upper bound for performance analysis of SM with generic digital signal modulation schemes. The accuracy of the analytical derivation has been verified via Monte Carlo simulations for a simple $M$-PSK modulation scheme and independent Nakagami- $m$ fading channels. Numerical results have shown that SM can offer better performance than conventional multiphase signal modulation schemes due to the efficient exploitation of the spatial domain for data modulation. Finally, a distributed architecture for the adoption of SM in the downlink of cellular networks has been proposed, and it has been shown that this approach may lead to high multiplexing gains since the number of antennas in the virtual MIMO system can be made arbitrary large without significantly affecting the complexity of the mobile handset. In fact, the complexity of the receiver increases only linearly with the number of antennas and the modulation order, even though ML-optimum decoding is used.

\section{ACKNOWLEDGMENT}

We gratefully acknowledge support from the EPSRC (EP/G011788/1) for this work. In addition, Harald Haas acknowledges the Scottish Funding Council support of his position within the Edinburgh Research Partnership in Engineering and Mathematics between the University of Edinburgh and Heriot Watt University.

\section{REFERENCES}

[1] J. Mietzner, R. Schober, L. Lampe, W. H. Gerstacker, and P. A. Höher, "Multiple-antenna techniques for wireless communications - A comprehensive literature survey", IEEE Commun. Surveys Tuts., vol. 11, no. 2, pp. 87-105, 2nd quarter 2009.

[2] P. Wolniansky, G. Foschini, G. Golden, and R. Valenzuela, "V-BLAST: An architecture for realizing very high data rates over the rich-scattering wireless channel", IEEE Int. Symposium on Signals, Systems, and Electronics, pp. 295-300, Sept./Oct. 1998.

[3] R. Y. Mesleh, H. Haas, S. Sinanovic, C. W. Ahn, and S. Yun, "Spatial modulation", IEEE Trans. Veh. Technol., vol. 57, no. 4, pp. 2228-2241, July 2008 .

[4] J. Jeganathan, A. Ghrayeb, and L. Szczecinski, "Spatial modulation: Optimal detection and performance analysis", IEEE Commun. Lett., vol. 12, no. 8, pp. 545-547, Aug. 2008.

[5] J. Jeganathan, A. Ghrayeb, L. Szczecinski, and A. Ceron, "Space shift keying modulation for MIMO channels", IEEE Trans. Wireless Commun., vol. 8, no. 7, pp. 3692-3703, July 2009.

[6] R. Y. Mesleh, M. Di Renzo, H. Haas, and P. M. Grant, "Trellis coded spatial modulation", IEEE Trans. Wireless Commun., Apr. 2010 (accepted, to appear).

[7] A. Alshamali and B. Quza, "Performance of spatial modulation in correlated and uncorrelated Nakagami fading channel", J. Commun., vol. 4, no. 3, pp. 170-174, Apr. 2009.

[8] M. Di Renzo and H. Haas, "On the performance of space shift keying MIMO systems over correlated Rician fading channels", ITG/IEEE Int. Workshop Smart Antennas, pp. 1-8, Feb. 2010.

[9] M. Di Renzo, R. Y. Mesleh, H. Haas, and P. Grant, "Upper bounds for the analysis of trellis coded spatial modulation over correlated fading channels", IEEE Veh. Technol. Conf., pp. 1-6, May 2010.

[10] M. Di Renzo and H. Haas, "A general framework for performance analysis of space shift keying (SSK) modulation for MISO correlated Nakagami- $m$ fading channels", IEEE Trans. Commun., Apr. 2010 (accepted, to appear)

[11] A. P. Prudnikov, Y. A. Brychkov, and O. I. Marichev, Integrals and Series. Vol. 3: More Special Functions, 2003.

[12] M. K. Simon and M.-S. Alouini, Digital Communication over Fading Channels: A Unified Approach to Performance Analysis, John Wiley \& Sons, Inc., 1st ed., 2000.

[13] H. L. Van Trees, Detection, Estimation, and Modulation Theory, Part I: Detection, Estimation, and Linear Modulation Theory, John Wiley \& Sons, Inc. 2001

[14] M. Di Renzo and H. Haas, "Performance comparison of different spatial modulation schemes in correlated fading channels", IEEE Int. Commun. Conf., pp. 1-6, May 2010.

[15] T. Weber, I. Maniatis, A. Sklavos, Y. Liu, E. Costa, H. Haas, and E. Schulz, "Joint transmission and detection integrated network (JOINT), a generic proposal for beyond $3 \mathrm{G}$ systems", Int. Conf. Telecommun., pp. 479-483, June 2002.

[16] M. Dohler, Virtual Antenna Arrays, Ph.D. Thesis, King's College London, London, UK, Nov. 2003.

[17] A. F. Molisch, P. V. Orlik, Z. Tao, R. Annavajjala, J. Zhang, L. Dong, and T. Kuze, "Base station cooperation", IEEE 802.16 Broadband Wireless Access WG, IEEE C802.16m-08/817, July 2008.

[18] C. Jandura, P. Marsch, A. Zoch, and G. P. Fettweis, "A testbed for cooperative multi cell algorithms", ACM Tridentcom, pp. 1-5, Mar. 2008.

[19] V. Jungnickel, L. Thiele, T. Wirth, T. Haustein, S. Schiffermüller, A. Forck, S. Wahls, S. Jäckel, S. Schubert, H. Gäbler, C. Juchems, F. Luhn, R. Zavrtak, H. Droste, G. Kadel, W. Kreher, J. Müller, W. Störmer, and G. Wannemacher, "Coordinated multipoint trials in the downlink", IEEE Broadband Wireless Access Workshop, pp. 1-7, Nov. 2009. 